\title{
4. EPHEMERIDES (EPHÉMÉRIDES)
}

PResident: G. A. Wilkins.

Vice-President: J. Kovalevsky.

Organizing Committee: G. M. Clemence, G. A. Chebotarev, R. L. Duncombe, W. Fricke.

\section{INTRODUCTION}

The appended reports of the directors of the national ephemeris offices provide a record of the current activity in the publication and preparation of astronomical ephemerides, including those published for use by navigators and surveyors and those prepared for astronomers whose special needs are not met in the printed almanacs. A brief review of the relevant work of other institutions and individuals is also given in the appendix. The reports also cover to some extent activities in positional astronomy and celestial mechanics (i.e. in the fields of interest of Commissions 7, 8, 17, 19,20 and 31). This report is therefore restricted to reviewing, firstly, the action that has been taken on matters raised at and since the 1967 meetings of the Commission and, secondly, the matters that will require attention at and after the 1970 meetings.

Before that review, it is appropriate to pay tribute to the memory of that almost legendary astronomer, Gaston Fayet, who died on 27 December 1967 at the advanced age of 93 . As member of the Bureau des Longitudes responsible for the production of the Connaissance des Temps from 1930 to 1961, and President of Commission 4 from 1938 to 1952, he contributed greatly to our subject, in addition to his researches in other fields particularly in respect of the minor planets.

\section{INTRODUCTION OF THE IAU SYSTEM OF ASTRONOMICAL CONSTANTS}

The changes in the printed ephemerides resulting from the adoption (a) in 1964 of the new IAU system of astronomical constants and (b) in 1967 of new bases for the solar part of the lunar theory and for the minor planet ephemerides will be virtually completed in the almanacs for 1972 . In all but a few cases the corrections have been incorporated into the main tabulations.

The problem of the epochs of the (ephemeris) time scales to which the solar and lunar ephemerides refer remains unresolved. The difficulties arise, firstly, from the discontinuity in the ephemeris of the apparent longitude of the Sun following the change in the constant of aberration and, secondly, from the suggestion that the solar and lunar ephemerides are referred to equinoxes that differ in right ascension by 1:34. The question therefore arises whether it would be desirable to bring the solar ephemeris into accord with the lunar ephemeris by introducing another discontinuity or whether it is sufficient merely to adjust the values of $\Delta T=$ E.T. - U.T. that are obtained from the comparisons between theory and observation. The latter appears to be preferable, but a clear statement of the basis of the adjustments is obviously required before such a procedure could be formally recommended.

\section{ASTRONOMICAL NAVIGATION}

The use of conventional astronomical methods for navigation and surveying shows little sign of extinction in spite of the increasing use of new methods based on radio transmissions, artificial satellites, and inertial navigation systems. Minor changes in the content and format of the various almanacs and auxiliary tables have been introduced and the process of worldwide standardization continues slowly. The introduction of a new system of international time signals (see below) will entail additional effort by the user if the full accuracy of astronomical techniques is to be utilised. 


\section{UNIT OF TIME}

The thirteenth General Conference on Weights and Measures adopted, on 13 October 1967, a resolution defining the unit of time in the International System (SI) of Units in terms of a transition frequency of caesium and abrogating the earlier resolutions defining the SI second in terms of ephemeris time. At present therefore, the responsibility for the definition and realization of the ephemeris time scale rests solely with the IAU, and Commission 4 should consider very carefully the future policy to be adopted in respect of the ephemeris time scale. In particular, consideration should be given to the possibility of adopting the SI second in place of the ephemeris second. This would once again make the coefficient of $T$ in the expression for the Sun's mean longitude subject to determination by observation. Such an approach would still leave open the question of the identity of the gravitational and atomic time scales; any fundamental difference between the two scales would give rise to apparent secular accelerations that could not be explained in terms of other known forces. The term ephemeris time could still be retained to provide a meaningful name for the identification of epochs determined by the comparison of observations and ephemerides rather than by 'clocks' (e.g. A.T.) or the rotation of the Earth (U.T. and s.T.).

\section{OTHER SI UNITS}

In recent years there has been a marked increase in the use of SI units in scientific literature, especially as many of the leading journals have recommended their use. Even apart from the question of the unit of time, there are three principal reasons why SI units should not be introduced into all astronomical ephemerides, and their auxiliary tables and explanations. Firstly, the use of the astronomical units of distance and mass in planetary dynamics allows distances and masses to be specified to a higher precision than would be possible if metres and kilogrammes were to be used; the significance of data expressed in astronomical units is also more easily understood. Secondly, ephemerides for practical uses must be designed so as to minimize both the effort and the risk of error associated with their use; this applies particularly to ephemerides for navigational purposes where the reductions must be made quickly, and often under adverse conditions. The unit of tabulation must therefore correspond to the units in which the measurements are made. In particular, the continued use of sexagesimal units for angle and time appears to be inevitable. Thirdly, in some circumstances it may be desirable, or even necessary, to specify some quantities in terms of the legal units of the country of publication.

With these reservations it seems desirable that SI units and conventions (e.g. restricting multiple and sub-multiple units to steps of one-thousand and one-thousandth, respectively) should be used in preference to, or in addition to, other units. It would be useful, however, if the Commission were to adopt a list indicating recommended usages in the field of dynamical astronomy so that the attempt to introduce a higher degree of standardization does not, in fact, have the opposite effect.

\section{INTERNATIONAL TIME SIGNALS}

Responsibility for the international coordination of the transmissions of time signals rests with the Consultative Committee on International Radio (CCIR). Following the adoption of the new definition of the SI second an international working group has recently recommended a change from the present system in which the primary transmissions are maintained to within one-tenth of a second of U.T. 2 by means of step adjustments in both frequency and epoch. If the recommendations are adopted, the primary transmissions will be based, as from the beginning of 1972, on a fixed frequency with respect to the SI second and on step adjustments of exactly 1 second. This implies that the departures from U.T.2 will exceed half-a-second and so will be much greater than the errors of human discrimination, such as in the timing of occultations and in the use of a sextant. The IAU has already expressed its willingness to accept such a change provided that certain conditions are met; these are intended to ensure that U.T. 2 will be readily available, even under field 
conditions, to a precision of 0.1 without danger of ambiguity. Such a change has no direct effect on the publication of astronomical ephemerides except in so far as it will be necessary to warn users about the departures from U.T.2 and to provide appropriate instructions for the application of corrections.

\section{INTERNATIONAL INFORMATION BUREAU ON ASTRONOMICAL EPHEMERIDES}

It was agreed at the 1967 meeting that the membership of the Commission's Working Group on Space Ephemerides should be enhanced by the co-option of other interested scientists. As a result, and at the invitation of the President of COSPAR, the Group met during the course of the COSPAR meeting in Prague in May 1969. After reviewing the current activities and requirements in the field of astronomical ephemerides, the Group considered how best it could facilitate both the exchange of ephemerides between the institutions engaged in preparing them and the supply of ephemerides to those other organisations that required them for research purposes. It was agreed to recommend that IAU and COSPAR should cooperate in setting up an information service that would provide information to the international scientific community on the availability of astronomical ephemerides, particularly those that exist in machine-readable form. Subsequently on the recommendation of Dr J. Kovalevsky, who is Chairman of COSPAR's Working Group I, it was agreed that the bureau should be sponsored by the IAU only, but with COSPAR represented on the advisory committee. The Commission will have to decide whether to adopt such a proposal.

\section{OBSERVATIONS OF THE POSITIONS OF THE MOON AND PLANETS}

The further improvement in the fundamental ephemerides of the Sun, Moon and planets depends on both the development of better theories and the comparison of the theories with observations to determine their arbitrary parameters. The Working Group on Space Ephemerides considered that, for the purposes of the proposed information bureau, astronomical ephemerides should be deemed to include collections of observations of the positions of planets and satellites. The Commission should consider whether it should, and could, do more to encourage and assist astronomers to prepare and make available collections of past observations and to make and publish new observations. (At the present time no Commission appears to cover adequately these activities for the Moon and major planets.) The task of locating, punching and, possibly, re-reducing past observations is so onerous that it would be a pity if there were to be unnecesary duplication in this valuable, but prosaic, activity. Further, the full rewards of the developments of new theories (often using much expensive computer time) will be lost if there is a lack of adequate observational material with which to compare the theories.

\section{COLLOQUIUM ON THE IAU SYSTEM OF ASTRONOMICAL CONSTANTS}

As a result of the discussions at the 1967 meeting and of subsequent correspondence it was decided to hold a Colloquium on the IAU System of Astronomical Constants at Heidelberg from 12 to 15 August 1970. The primary purpose of the meeting is to provide the bases for subsequent formal proposals for the adoption or otherwise of new values of those astronomical constants that were left unchanged when the IAU system was adopted in 1964. The principal constants concerned are the precessional constants and the masses of the planets. It is necessary to consider not only the strength of the evidence for more accurate values, but also the possible procedures for, and consequences of, the introduction of new values. At the meetings of the Commission at Brighton it will be necessary to decide what action should be taken on the recommendations of the Colloquium. The present consensus of opinion seems to be that, if it is decided to adopt new values, the formal resolution should be considered at the General Assembly in 1973.

The question of the year of introduction into the almanacs of the use of the equinox of $2000 \cdot 0$ has been raised by Dr J. Kovalevsky. Only a small number of comments have so far been received, 
but the majority of them are strongly in favour of making no decision until the question of the change in the precessional constants has been settled, with the possibility of introducing both changes into the ephemerides for 1980 . It has also been suggested (a) that the preparation of highprecision star catalogues referred to the new equinox should similarly be deferred, and (b) that the E-terms of aberration should be removed from the mean places so as to simplify rigorous reductions to and from apparent places.

\section{FUTURE PUBLICATION OF FUNDAMENTAL EPHEMERIDES}

As previous Presidents have implied the national almanacs are becoming less suitable for the publication of fundamental ephemerides, whose primary purpose is to provide internationally recognized standards with which observations and new theories can be compared. This arises partly from the increasing use of computers in the preparation of ephemerides and in the reduction of observations, and partly from the introduction of new techniques of observation in which quantities other than apparent right ascension and declination are measured. Further, the present system necessarily entails a time lag of between five and eight years before an improved ephemeris can be introduced into the main tabulations printed in the almanacs. This is now quite unsatisfactory since it means that in practice other ephemerides are used; the Commission should therefore consider alternative procedures.

The principal desiderata for a fundamental ephemeris are: firstly, that it should cover a period of many years and be available in both printed and machine-readable form; and, secondly, that it should be precisely computed on a recognized and clearly-defined basis. These conditions are often better met by an independent publication (such as the ephemerides in the Astronomical Papers of the American Ephemeris) than by inclusion in an annual publication, which has also to provide ephemerides for other purposes.

It is therefore suggested that in future the fundamental ephemerides should first be published in independent form covering many years, using computer-based techniques so as to reduce the cost and effort required. The task of the Commission would then be to decide when to recognize a new ephemeris as an international standard. The final decision need be taken only after the ephemeris has been published and scrutinized to establish the quality of its numerical values and documentation, although naturally there would usually be prior consultation. The Commission could assign a suitable reference code (such as is now adopted for the various forms of the lunar ephemeris) so as to facilitate the specification of the particular ephemeris used in a comparison between observation and theory. The Commission could recognize both heliocentric and geocentric ephemerides as well as special ephemerides, such as an ephemeris of the rectangular coordinates of the geocentre with respect to the centre of mass of the solar system for use in the reduction of observations of pulsars, etc. The adoption of such a system would allow greater freedom in the design of the almanacs to meet the day-to-day requirements of the users.

\section{CONCLUSIONS}

The period of change that started with the proposal in 1950 for the introduction of ephemeris time is not yet over, but in the next few years we may hope to consolidate the progress that has been made since 1950 and provide a firm foundation for dynamical astronomy during the remainder of the twentieth century. 